\title{
Distinguished Dissertations
}

Springer

London

Berlin

Heidelberg

New York

Barcelona

Hong Kong

Milan

Paris

Singapore

Tokyo 
Other titles published in this Series:

Search and Planning Under Incomplete Information: A Study Using Bridge Card Play Ian Frank

Theorem Proving with the Real Numbers

John Harrison

Hardware Evolution: Automatic Design of Electronic Circuits in Reconfigurable Hardware by Artificial Evolution

Adrian Thompson

User-Developer Coooperation in Software Development

Eamonn O’Neil

A Combination of Geometry Theorem Proving and Nonstandard Analysis, with Application to Newton's Principia

Jacques Fleuriot

Accurate Visual Metrology from Single and Multiple Uncalibrated Images

Antonio Criminisi

Asynchronous System-on-Chip Interconnect

John Bainbridge 
John MacCormick

Stochastic Algorithms

for Visual Tracking

Probabilistic Modelling and Stochastic Algorithms for Visual Localisation and Tracking 
John MacCormick

Systems Research Center, Compaq Computer Corporation, 130 Lytton Avenue, Palo Alto, CA 94301-1044, USA

\title{
Series Editor
}

Professor C.J. van Rijsbergen

Department of Computing Science, University of Glasgow, G12 8RZ, UK

\author{
British Library Cataloguing in Publication Data \\ MacCormick, John \\ Stochastic algorithms for visual tracking. - (Distinguished \\ dissertations) \\ 1.Computer vision - Mathematical models 2. Algorithms \\ 3.Stochastic processes \\ I.Title \\ 006.3'7 \\ ISBN-13: 978-1-4471-1176-4 \\ e-ISBN-13: 978-1-4471-0679-1 \\ DOI: $10.1007 / 978-1-4471-0679-1$ \\ Library of Congress Cataloging-in-Publication Data \\ A catalog record for this book is available from the Library of Congress.
}

Apart from any fair dealing for the purposes of research or private study, or criticism or review, as permitted under the Copyright, Designs and Patents Act 1988, this publication may only be reproduced, stored or transmitted, in any form or by any means, with the prior permission in writing of the publishers, or in the case of reprographic reproduction in accordance with the terms of licences issued by the Copyright Licensing Agency. Enquiries concerning reproduction outside those terms should be sent to the publishers.

Distinguished Dissertations ISSN 1439-9768

ISBN-13: 978-1-4471-1176-4 Springer-Verlag London Berlin Heidelberg

a member of BertelsmannSpringer Science+Business Media GmbH

http://www.springer.co.uk

\section{(c) Springer-Verlag London Limited 2002}

Softcover reprint of the hardcover 1st edition 2002

The use of registered names, trademarks etc. in this publication does not imply, even in the absence of a specific statement, that such names are exempt from the relevant laws and regulations and therefore free for general use.

The publisher makes no representation, express or implied, with regard to the accuracy of the information contained in this book and cannot accept any legal responsibility or liability for any errors or omissions that may be made.

Typesetting: Camera-ready by author 


\section{Preface}

Computer vision is hard. One way to make progress on hard problems is to develop a coherent mathematical theory which describes them. This book develops one such theory for the field of visual tracking, using probabilistic models and stochastic algorithms. In an applied science like computer vision, however, theory is of little use without practice. Hence, the book also demonstrates the use of this theory in real visual tracking systems.

The stochastic algorithms employed here - variously known as "particle filters", or the "Condensation algorithm" - are introduced in Chapter 2 via a formulation based on sequences of weighted particles. The properties of the particle filter are proved rigorously, yet presented in a manner intended to be accessible to a general computer vision audience. Chapter 3 introduces the other essential ingredient for visual tracking: a set of probabilistic models for object localisation. These models, called "contour likelihoods", are based on the statistical properties of image features. Chapter 4 explains the use of contour likelihoods in localisation and tracking problems, and Chapter 5 uses Markov random fields to generalise the contour likelihood for modelling the occlusion of foreground objects. Contour likelihoods can also be derived for collections of more than one object. Chapter 6 shows how such derivations lead to an "exclusion principle" which enables the successful tracking of foreground objects which occlude each other. Finally, Chapter 7 introduces the technique of "partitioned sampling", which dramatically reduces the computational expense of particle filters in certain problems. As in the previous chapters, I have tried to prove the results in a rigorous yet accessible manner. Readers equipped with a basic knowledge of mathematics, engineering, or computer science will have no difficulty in following the text.

This book arose from my Ph.D. thesis at the University of Oxford. My greatest debt is to Andrew Blake, who was my Ph.D. supervisor; he provided many ideas and much insight. I am also indebted to my colleagues at the Oxford Visual Dynamics Laboratory during the late 1990s: Benedicte Bascle, Colin Davidson, Jonathan Deutscher, Michael Isard, Bob Kaucic, Ben North, Jens Rittscher, Josephine Sullivan, and Andrew Wildenberg. Michael deserves a special vote of thanks because he wrote a lot of the code that I used and was also a very good friend. Many other people helped to keep me sane or were nice to me even when they realised they were failing in this goal; among them are Bear and Christie Bolton, Paul Harvey and his family, Andy Marsham, Jon Scammon, and everyone from OUHC. 
For financial support, I am very grateful to the trustees of the Eliot Davis Memorial Scholarship, the trustees of the Jowett Scholarship, and the European Union IMPROOFS grant. Three Oxford colleges - Exeter, Balliol, and Linacre - have assisted me, and I am grateful to each for its generosity. The Systems Research Center of Compaq Computer Corporation supported me during the preparation of the manuscript, and the British Computer Society enabled its publication.

My family all live far away but are close in other ways. In fact they probably helped out much more than they realised, and I would especially like to thank Mum, Dad, Andrew, Jude and G\&G, P\&B, JGJS\&A, RLVSK\&L. The book is dedicated to my parents, with love and respect.

John MacCormick

Palo Alto, California

January, 2002 


\section{Contents}

1 Introduction and background 1

1.1 Overview .................... 2

1.2 Active contours for visual tracking $\ldots \ldots \ldots \ldots \ldots \ldots$

1.2.1 Splines and shape space . . . . . . . . . . . . 4 .

1.2.2 Dynamical models using auto-regressive processes . . . 5

1.2.3 Measurement methodology . . . . . . . . . . 6

2 The Condensation algorithm $\quad 8$

2.1 The basic idea . . . . . . . . . . . . . . 8

2.2 Formal definitions $\ldots \ldots \ldots \ldots \ldots \ldots \ldots$

2.2.1 Technical detail: convergence of distribution-valued distributions . . . . . . . . . . . . . . . . . 14

2.2.2 The crucial definition: how a particle set represents a distribution . . . . . . . . . . . 15

2.3 Operations on particle sets $\ldots \ldots \ldots \ldots \ldots$

2.3.1 Multiplication by a function . . . . . . . . . . . . 17

2.3.2 Applying dynamics . . . . . . . . . . . . . . . . . 18

2.3.3 Resampling . . . . . . . . . . . . . . . . . 20

2.4 The Condensation theorem . . . . . . . . . . . . . 24

2.5 The relation to factored sampling, or "where did the proof go?" 25

2.6 "Good" particle sets and the effective sample size . . . . . . 26

2.6.1 The survival diagnostic . . . . . . . . . . . . 28

2.6.2 From effective sample size to survival diagnostic . . . . 31

2.6.3 Estimating the weight normalisation . . . . . . . . 33

2.6.4 Effective sample size of a resampled set $\ldots \ldots \ldots . . .33$

2.7 A brief history of Condensation . . . . . . . . . . . 35

2.8 Some alternatives to Condensation . . . . . . . . . . 37

3 Contour likelihoods 38

3.1 A generative model for image features $\ldots \ldots \ldots \ldots \ldots$

3.1.1 The generic contour likelihood . . . . . . . . . 42

3.1.2 The Poisson likelihood . . . . . . . . . . . . . . . 45

3.1.3 The interior-exterior likelihood . . . . . . . . . 46

3.1.4 The order statistic likelihood . . . . . . . . . . . 49

3.1.5 The contour likelihood ratio . . . . . . . . . . . 50

3.1.6 Results and examples . . . . . . . . . . . . 51 
3.2 Background models and the selection of measurement lines . . 54

3.2.1 Discussion of the background model . . . . . . . . 55

3.2.2 Independence of measurement lines . . . . . . . . . 55

3.2.3 Selection of measurement lines . . . . . . . . . 57

3.3 A continuous analogue of the contour likelihood ratio . . . . . 60

3.3.1 The continuous model . . . . . . . . . . . . 60

3.3.2 Likelihoods for $H_{O}$ and $H_{B} \ldots \ldots \ldots \ldots \ldots 6$

3.3.3 Problems with the continuous ARP model . . . . . . 63

4 Object localisation and tracking with contour likelihoods 65

4.1 A brief survey of object localisation . . . . . . . . . 65

4.2 Object localisation by factored sampling . . . . . . . . 68

4.2 .1 Results . . . . . . . . . . . . . . 70

4.2.2 Interpretation of the gradient threshold $\ldots \ldots \ldots 73$

4.3 Estimating the number of targets . . . . . . . . . 75

4.4 Learning the prior . . . . . . . . . . . . . 79

4.5 Random sampling: some traps for the unwary . . . . . . . . 80

4.6 Tracker initialisation by factored sampling . . . . . . . . . 84

4.6.1 Kalman filter tracker . . . . . . . . . . . . . . . 85

4.6.2 The Condensation tracker . . . . . . . . . . 85

4.7 Tracking using Condensation and the contour likelihoods . . . . 86

4.7.1 The robustified colour contour likelihood . . . . . . . 86

4.7.2 Implementation of a head tracker . . . . . . . . . 89

5 Modelling occlusions using the Markov likelihood 92

5.1 Detecting occluded objects . . . . . . . . . . . . . 92

5.2 The problem with the independence assumption $\ldots \ldots \ldots 94$

5.3 The Markov generative model . . . . . . . . . . . . . 95

5.4 Prior for occlusions . . . . . . . . . . . . . . . 96

5.5 Realistic assessment of multiple targets . . . . . . . . . . . 99

5.5.1 Explanation of results . . . . . . . . . . . . . 99

5.5 .2 Experimental details . . . . . . . . . . . . . 100

5.6 Improved discrimination with a single target . . . . . . . . 102

5.7 Faster convergence using importance sampling . . . . . . . 103

5.8 Random samples using MCMC . . . . . . . . . . . . . 107

5.9 Calculating the partition functions . . . . . . . . . . . 109

5.10 Further remarks . . . . . . . . . . . . . . . 110

6 A probabilistic exclusion principle for multiple objects 112

6.1 Introduction . . . . . . . . . . . . . . . . . . 112

6.2 A generative model with an exclusion principle . . . . . . . 114

6.2.1 Description of the generative model . . . . . . . . . 114

6.2.2 Likelihoods derived from the generative model . . . . . 115

6.2.3 Where does the "exclusion principle" come from? . . . . 116

6.2.4 The full likelihood . . . . . . . . . . . . . . . . . . 118

6.3 Tracking multiple wire-frame objects . . . . . . . . . 118 
6.4 Tracking multiple opaque objects . . . . . . . . . . . . . 119

7 Partitioned sampling $\quad 124$

7.1 The need for partitioned sampling . . . . . . . . . . 124

7.2 Weighted resampling . . . . . . . . . . . . . . . 127

7.3 Basic partitioned sampling . . . . . . . . . . . . 130

7.4 Branched partitioned sampling . . . . . . . . . . . 131

7.5 Performance of partitioned sampling . . . . . . . . . 133

7.6 Partitioned sampling for articulated objects . . . . . . . . . . 134

7.6.1 Results: a vision-based drawing package . . . . . . . 138

8 Conclusion? 144

$\begin{array}{ll}\text { Appendix A } & 146\end{array}$

A.1 Measures and metrics on the configuration space . . . . . . 146

A.2 Proof of the interior-exterior likelihood . . . . . . . . . . 147

A.3 Del Moral's resampling lemma and its consequences . . . . . 149

$\begin{array}{ll}\text { Appendix B } & 153\end{array}$

B.1 Summary of notation . . . . . . . . . . . 153

$\begin{array}{ll}\text { Bibliography } & 155\end{array}$

$\begin{array}{ll}\text { Index } & 165\end{array}$ 